\title{
Baylisascaris sp. infection in a pet kinkajou Potos flavus
}

\author{
K. TAIRA ${ }^{*}$, Y. UNE ${ }^{2}$, V. ŠNÁBEL ${ }^{3}$, H. SUGIYAMA ${ }^{4}$
}

\begin{abstract}
${ }^{1}$ Laboratory of Parasitology, School of Veterinary Medicine, Azabu University, 1-17-71 Fuchinobe, Chuo-ku, Sagamihara, Kanagawa 252-5201, Japan, E-mail: taira@azabu-u.ac.jp; ${ }^{2}$ Laboratory of Pathology, School of Veterinary Medicine, Azabu University, 1-17-71 Fuchinobe, Chuo-ku, Sagamihara, Kanagawa 252-5201, Japan; ${ }^{3}$ Institute of Parasitology, Slovak Academy of Sciences, 04001 Košice, Slovakia; ${ }^{4}$ Department of Parasitology, National Institute of Infectious Disease, 1-23-1 Toyama, Shinjuku, Tokyo 162-8640, Japan
\end{abstract}

\begin{abstract}
Summary
The nematodes of genus Baylisascaris are common intestinal roundworms of carnivores such as raccoons, skunks, badgers, martens and bears. This report describes Baylisascaris sp. infection in a pet kinkajou Potos flavus imported into Japan from Guyana. Nematode eggs were detected in feces of the juvenile kinkajou in 2011 during a routine veterinary examination. A sequence analysis of the ITS2 nuclear target clustered the examined isolate with B. procyonis and $B$. columnaris, with $7.8-8.8 \%$ base differences from these taxa. Eleven tandem G-A repeats identified in the polymorphic repetitive region further differentiate the kinkajou's roundworm from recognized Baylisascaris species. This classified the studied isolate as referring to Baylisascaris sp., with its precise species delineation remaining to be determined. Given that the Baylisascaris sp. from the kinkajou is genetically closely affiliated with $B$. procyonis having a serious disease-producing capacity, the report appeals for precautions in informing people to avoid transmission risk.
\end{abstract}

Keywords: Baylisascaris sp.; Potos flavus; ITS2; zoonosis

\section{Introduction}

The nematodes of genus Baylisascaris are intestinal roundworms of carnivores such as raccoons, skunks, badgers, martens and bears, and their larvae migrate in a range of small animal paratenic hosts serving as prey (Kazacos et al., 2001; Gavin et al., 2005). The most pathogenic Baylisascaris species is B. procyonis, which is indigenous in North American raccoons (Procyon lotor) and has been demonstrated to occur in raccoons introduced around the world. The high prevalence of $B$. procyonis infection has been reported especially in wild raccoons in Germany and those kept in zoos in Japan (Miyashita, 1993; Sato et al., 2001; Bauer, 2011). The larvae of these nematodes cause ocular and neural larva migrans in humans, and infection can potentially result in serious encephalitis, with permanent deficits or even death (Kazacos et al., 2011).

The kinkajou Potos flavus has also been reported to be a definitive host of $B$. procyonis, which is identified based on morphological observations (Overstreet, 1970). In December 2011 , a routine veterinary examination of a juvenile male pet kinkajou that had been caught in nature, imported from Guyana to Japan and purchased from an exotic pet shop in the same month, revealed ascarid eggs in the feces. The kinkajou was orally administrated once with an anthelmintic containing $72 \mathrm{mg}$ of pyrantel, $75 \mathrm{mg}$ of febantel and $25 \mathrm{mg}$ of praziquantel (Drontal ${ }^{\circledR}$ Plus, Bayer). One day after the treatment, a nematode body fragment was expelled in the feces. No eggs had been detected in feces after the treatment.

This report describes finding of Baylisascaris sp. in a pet kinkajou in Japan, which was analyzed using the second internal transcribed spacer (ITS2) rDNA sequence, and outlines the potential risk of human infection with Baylisascaris sp. associated with the keeping of kinkajous as pets.

\section{Materials and methods}

\section{Parasite material}

A fragment of a nematode body measuring approximately $20 \mathrm{~mm}$-long and $2 \mathrm{~mm}$-wide was obtained from feces of a pet kinkajou kept in Kanagawa Prefecture, Japan. The samples were preserved in $5 \mathrm{ml}$ of $10 \%$ formalin solution for 2 days. No intact worm bodies or posterior or anterior ends of the worms were obtained.

\section{Fecal examination}

To detect parasite eggs, the fecal debris of the kinkajou $(<0.5 \mathrm{~g})$ was examined by the flotation method using a saturated $\mathrm{NaCl}$ solution containing glucose (specific gravity of 1.27) (Roepstorff \& Nansen, 1998). The dimensions of the obtained eggs $(n=20)$ were thereafter measured. 
Molecular procedures

The nematode tissue sample was washed with TE buffer ( $\mathrm{pH}$ 8.0) solution and then incubated in the buffer overnight. Genomic DNA was extracted from the tissue using a QIAamp DNA Mini Kit (Qiagen, Tokyo, Japan) according to the manufacturer's instructions, with several modifications (Šnábel et al., 2012). The DNA extract was used as template for amplification of the second internal transcribed spacer (ITS2) region of ribosomal DNA by PCR. The forward primer LC1 (5'-CGACTATCGATGAAGAA CGCAGC-3') and the reverse primer HC2 (5'-ATATGCT TAAGTTCAGCGGG-3'), which corresponded to the conserved 3' and 5' ends of the 5.8S-ITS2-28S regions, were used. Primers were formerly designed by Ellis et al., (1986) and Qu et al. (1988), and were simultaneously used in a phylogenetic study on schistosomes undertaken by Despres et al. (1992). The PCR procedure was performed using the following protocol: $94{ }^{\circ} \mathrm{C}$ for $12 \mathrm{~min}$ (polymerase activation), followed by 30 cycles of denaturation at $94{ }^{\circ} \mathrm{C}$ for $30 \mathrm{sec}$, annealing at $58{ }^{\circ} \mathrm{C}$ for $45 \mathrm{sec}$, extension at $72{ }^{\circ} \mathrm{C}$ for $45 \mathrm{sec}$, and a final extension step of $72{ }^{\circ} \mathrm{C}$ for $7 \mathrm{~min}$. The amplicons $(2 \mu \mathrm{l})$ were examined by $2 \%$ ethidium bromide-stained agarose gel electrophoresis to assess the amplification efficiency. The selected PCR products were then purified and sequenced in both directions using an ABI3730XL automated sequencer (Life Technologies, CA). To seek nematodes with the closest genetic relatedness to the examined sample, the sequence homology was searched using the Basic Local Alignment Search Tool (BLAST 2.2.22) tool. The 5' and 3' ends of the ITS2 sequence commonly determined for $B$. procyonis were verified by comparison with the consensus sequences for re- lated ascarid isolates stored in GenBank, namely Baylisas-

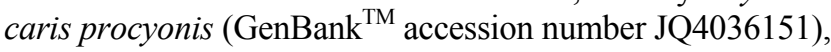
$B$. columnaris genotype 1 (KC543487), B. columnaris genotype 2 (KC543486), B. transfuga (HM594951), B. schroederi (JN210912), Ascaris suum (AB571302), A. lumbricoides (AB571298), Parascaris equorum (JN617987), T. canis (JF837169), Toxocara vitulorum (FJ418784), T. cati (AB571303), and Toxascaris leonina (JF837174).

These reference sequences were then aligned with the sequence obtained in the present study using ClustalW software (Thompson et al., 1994). To indicate the phylogenetic position of nematode from the pet kinkajou and to evaluate evolutionary relationships within the group of ascarid nematodes, the compromised sequences trimming to equal lengths were subjected to clustering analysis using the neighbor-joining (NJ) and maximum likelihood (ML) clustering methods with the Kimura 2-parameter calculated for a distance matrix. Caenorhabditis elegans (JN636101) was used as an out-group. Bootstrap analysis with 1,000 replicates was performed to assess tree robustness using the MEGA 5 program (Tamura et al., 2011). The sequence of the kinkajou isolate was deposited in GenBank with the accession number KF680774.

\section{Results}

Eggs detected in the feces of a pet kinkajou were ellipsoidal in shape, brown in color, and contained large unicellular embryo with a thick shell and roughly granular surface (Fig. 1). The eggs measured $76.8 \pm 2.26 \mu \mathrm{m}(\mathrm{n}=20$, mean $\pm \mathrm{SD}$; range: $73-81 \mu \mathrm{m}$ ) in the long axis and $64.4 \pm 1.02$ $\mu \mathrm{m}$ (range: $62.9-66.5 \mu \mathrm{m}$ ) in the transverse axis.

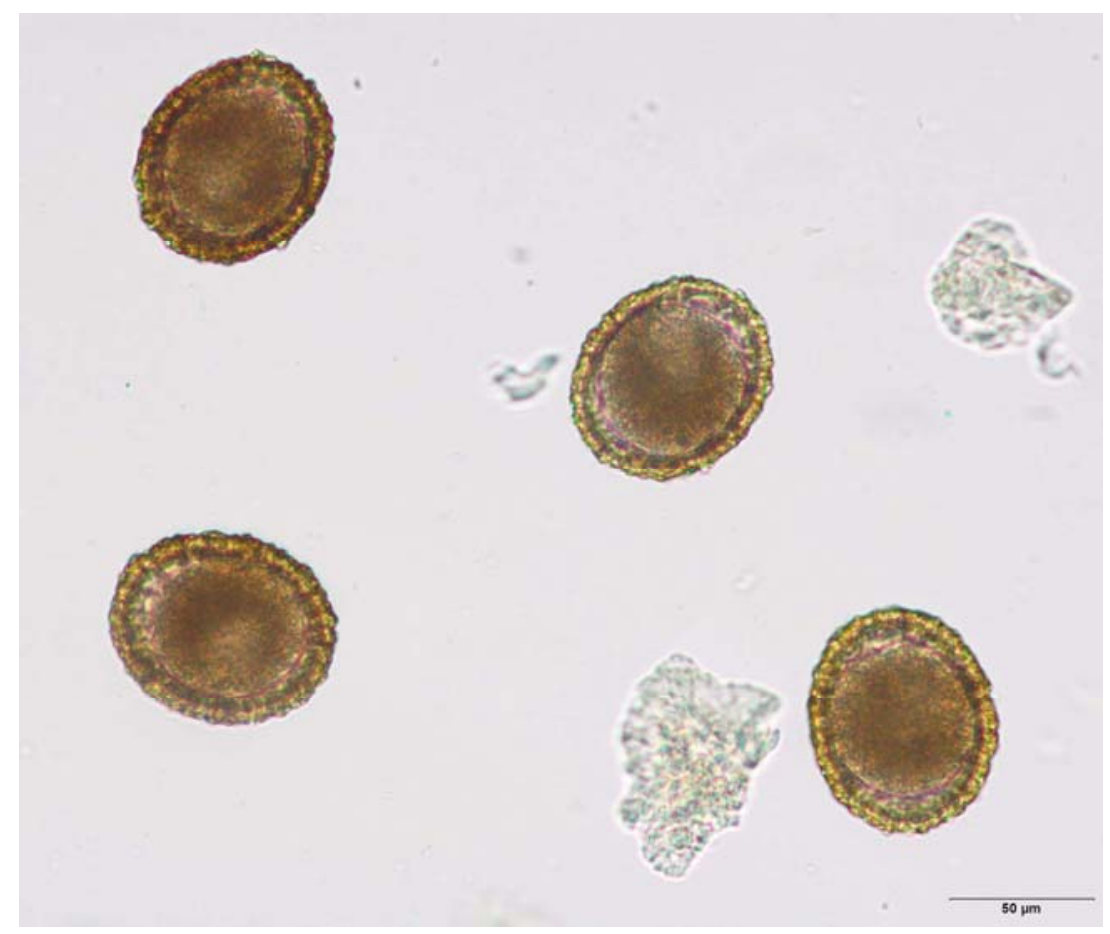

Fig. 1. Nematode eggs detected in feces of a pet kinkajou by the flotation method using a saturated NaCl solution containing glucose (1.27 SG); Eggs measured $76.8 \pm 2.26 \mu \mathrm{m}(\mathrm{n}=20$, mean $\pm \mathrm{SD}$; range: $73-81 \mu \mathrm{m})$ in the long axis and $64.4 \pm 1.02 \mu \mathrm{m}(\mathrm{range}: 62.9-66.5 \mu \mathrm{m})$ in the transverse axis 


\section{JP1}

BP (JQ403615)

BC1 (KC543487)

BC2 (KC543486)

\section{JP1 \\ BP (JQ403615) \\ BC1 (KC543487) \\ BC2 (KC543486)}

\author{
JP1 \\ BP (JQ403615) \\ BC1 (KC543487) \\ $\mathrm{BC} 2$ (KC543486)
}

\author{
JP1 \\ BP (JQ403615) \\ $\mathrm{BC} 1$ (KC543487) \\ BC2 (KC543486)
}

\section{JP1}

BP (JQ403615)

BC1 (KC543487)

BC2 (KC543486)

\section{JP1}

BP (JQ403615)

BC1 (KC543487)

BC2 (KC434486)

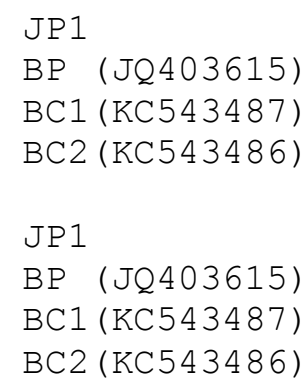

$$
\begin{aligned}
& \text { JP1 } \\
& \text { BP (JQ403615) } \\
& \text { BC1 (KC543487) } \\
& \text { BC2 (KC543486) }
\end{aligned}
$$

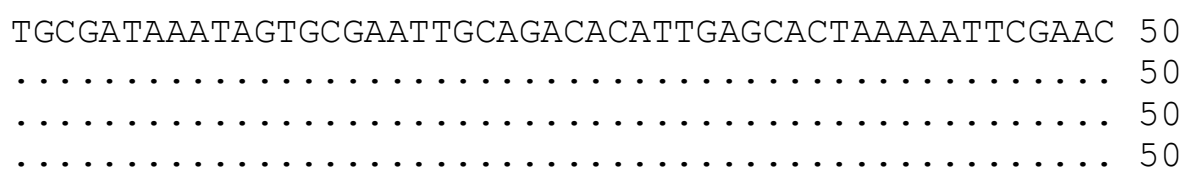

GCACATTGCGCCATCGGGTTCATTCCCGTTGGCACGTCTGGCTGAGGGTT 100

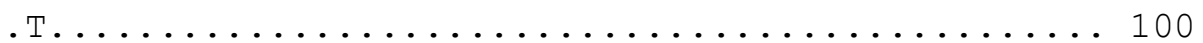

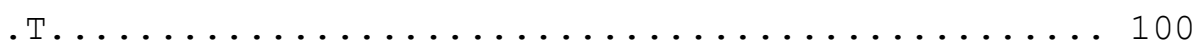

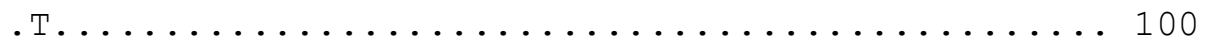

GAAATATCGTAAgAАTGCCATTTATGAATTTCAATATGGCATATTCGA 150

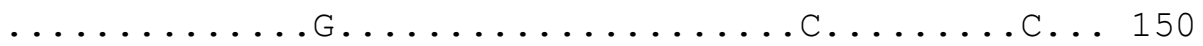

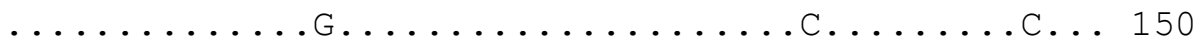

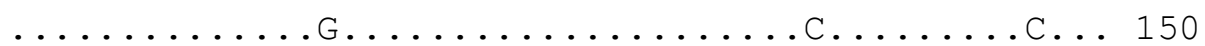

TAAGCTATGgTGgTAgACGAATAAAAGAAgTACTATCGTACCTTCTTCAG 200

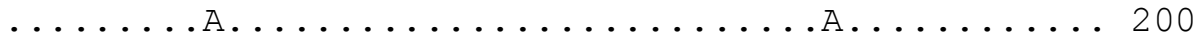

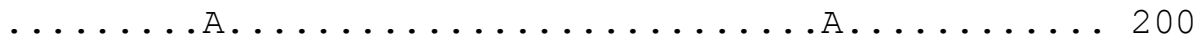

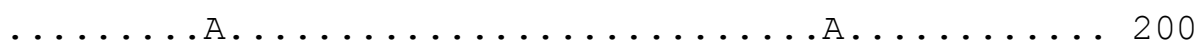

CAтAтAтGATGCAATAACTCGTTCTCATTTGCTTCGACGAGCTCAGAGAG 250

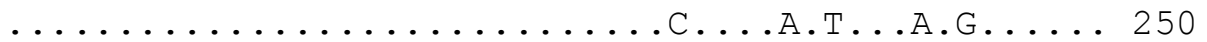

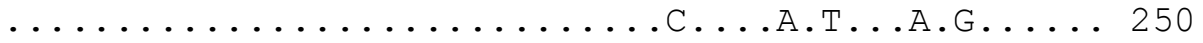

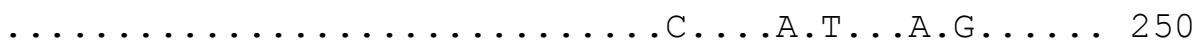

AGAGA GA GA GAGAGAGAAAGAGAAGAAAGAAGAAAGAGAAAGAATATA 300

AGAGAGAGAGAGA--------------------AAGAGAAAGAATATA 278

AGAGAGAGA----------------------AAGAGAAAGAATATA 274

AGAGAGA------------------------AAGAGAAAGAATATA 272

TGCATCAAGAAATATCGTGTCGCTCTTAAAAATCGATTCCAGCGTATAT 350

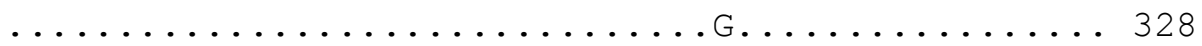

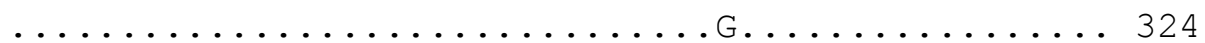

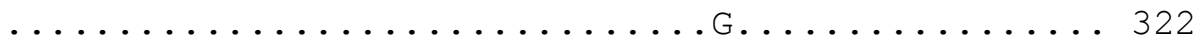

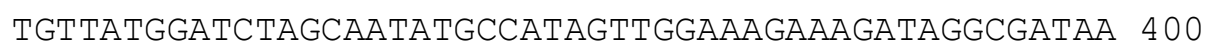

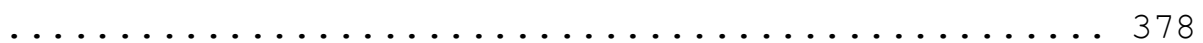

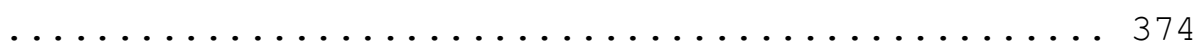

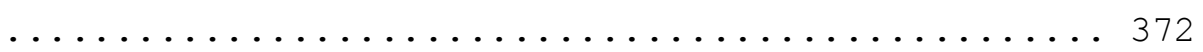

TGATGCATATAAAGGATTTTTTGACCTCAGCTCA 434

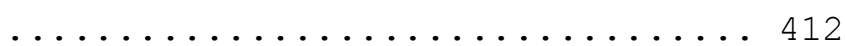

$\ldots \ldots \ldots \ldots \ldots \ldots \ldots \ldots$

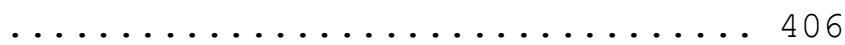

Fig. 2. Polymorphic sites in ITS2 sequences (434 bp) of JP1 kinkajou isolate related to Baylisascaris procyonis (BP);

B. columnaris, genotype 1 (BC1); B. columnaris, genotype 2 (BC2). Dots indicate identity with reference (JP1) sequence

The PCR product in the interpretable length of $434 \mathrm{bp}$ was yielded by amplification of the rDNA region spanning the 3 ' end of the 5.8S, ITS2, and 5' end of 28S rDNA from the worm fragment. BLAST homology analysis revealed that the closest phylogenetic relatives to the nematode in the present study; herein denoted as JP1 isolate, are B. procyonis and $B$. columnaris among present GenBank data. $B$. procyonis isolate from raccoon in Norway (JQ403615) and two genotypes of $B$. columnaris expelled from skunks in
Netherlands (KC543486, KC543487) were taken as references for analyses of variations in nucleotide composition. Pairwise comparison showed that the JP1 isolate differed in $7.8 \%$ of bases from B. procyonis by manifesting 22 insertions and 12 nucleotide substitutions, and in $8.3 \%$ $8.8 \%$ of bases from the two $B$. columnaris genotypes by manifesting $24-26$ insertions and 12 nucleotide substitutions (Fig. 2).

A substantial part of variation differentiating the kinkajou 


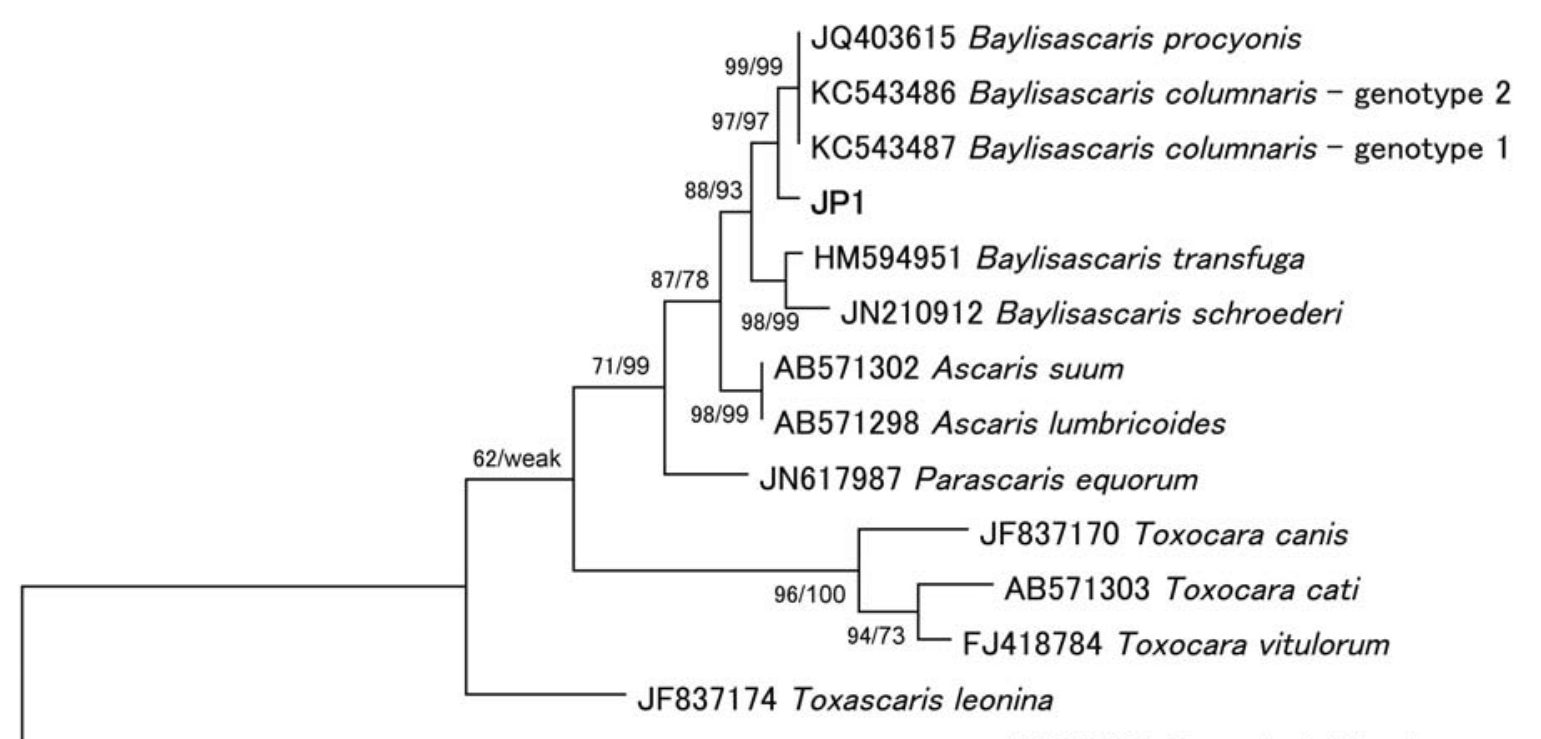

JN636101 Caenorhabditis elegans

0.1

Fig. 3. Dendrogram constructed by neighbor-joining (NJ) and maximum likelihood (ML) methods based on ITS2 nuclear sequences of Baylisascaris and related ascarid species; JP1 isolate was examined in the present study; Numbers at the nodes of branches refer to bootstrap values

( 1,000 pseudoreplications) derived from NJ (first value) and ML analyses. Weak bootstrap support was indicated at values $<60 \%$

isolate JP1 from the related species is attributed to $22-26$ insertions from positions 264 upwards, partially consisting of G-A tandem repeats, the number of which consistently distinguishes Baylisascaris species. For nucleotide substitutions, the most polymorphic region for JP1 isolate, comprising 5 of 12 base exchanges, was located between positions 231 - 244 of ITS2 (Fig. 2). Among recorded nucleotide exchanges relative to $B$. procyonis, 9 were transitions and 3 transversions. Resulting transition/transversion (ts/tv) ratio 3:1 for these taxa is generally assumed for closely related organisms.

Phylogenetic trees constructed by NL and ML analyses of ITS2 sequences of the 11 ascarid species revealed the similar topology, with two large clades separating Ascarididae and Toxocaridae isolates. The analyzed JP1 isolate was located in a well-supported subclade (bootstrap $88 \%$ in NJ, $93 \%$ in ML) consisting of Baylisascaris representatives, with the two clusters being inferred for this genus. The JP1 isolate has grouped together with $B$. procyonis and $B$. columnaris, with some discrimination from these taxa (Fig. 3). From remaining Baylisascaris species, B. transfuga and $B$. schroederi located in second cluster, the JP1 isolate differed in $14.3 \%$ and $15.4 \%$ of ITS bases, respectively.

\section{Discussion}

In the present study, a peculiar genetic composition of a Baylisascaris isolate from a pet kinkajou imported to Japan from South America, consistently differing from the closest B. procyonis with public health relevance, was recorded.
The ascarid eggs detected in feces from the kinkajou were morphologically similar to those of $B$. procyonis (raccoon roundworm), with eggs measuring 63-88 $\mu \mathrm{m}$ (mean: 68-76 $\mu \mathrm{m})$ by $50-70 \mu \mathrm{m}$ (mean: $55-61 \mu \mathrm{m}$ ), and $B$. columnaris (skunk roundworm) with eggs measuring $72.5 \pm 4.1 \mu \mathrm{m}$ by $63.2 \pm 7.5 \mu \mathrm{m}$ (Kazacos et al., 2011; Franssen et al., 2013). Slightly closer values with regard to the JP1 isolate were thus associated with $B$. columnaris, particularly in the length of transverse axis $(64.4 \pm 1.02 \mu \mathrm{m}$ in JP1 isolate). Nevertheless, morphometric identification in Baylisascaris is often hampered by diversity in size and developmental stages that makes the unequivocal recognition of several species including $B$. procyonis and B. columnaris problematic (Berry, 1985; Franssen et al., 2013).

The nuclear rDNA internal transcribed spacer is extensively being used for phylogenetic reconstruction and distinguishing between closely related species, including ascarids (Zhu et al., 2001; Pawar et al., 2012). In the present study, a unique divergence pattern was derived from the sequences of the ITS2 region for the kinkajou isolate suggesting that the nematode could not be entirely allocated to the closest recognized Baylisascaris species. Molecular prospecting as a tool for search and evaluation of the existence of separate taxa within the morphospecies has been often addressed to more clarify systematics in ascarid nematodes (Nadler \& Huspeth, 2000; Derycke et al., 2010). As Blouin (2002) summarized, the level of fixed sequence difference in ITS between closely related nematodes is relatively frequently $\leq 1 \%$, unlike mitochondrial DNA, in which helminth species typically differ in at least $10 \%$ of sequences (Vilas et al., 2005). The $7.8-8.8 \%$ 
range of nucleotide difference between the JP1 isolate and the closest retrieved sequences of $B$. procyonis and $B$. columnaris in ITS2 therefore classified the isolate from the pet kinkajou as referring to Baylisascaris sp., with its precise species categorization remaining to be determined. Another question emerges whether or not the present isolate pertains to any described Baylisascaris spp., for which sequencing data are not yet available in GenBank. The genus currently contains eight recognized species (Kazakos, 2013). From these, apart from 4 species with available nuclear sequences, transmissions of $B$. melis linked to badgers absent in South America, and B. tasmaniensis with marsupial carnivore hosts endemic for Tasmania, exclude these taxa as plausible for infesting kinkajou in Guyana. From remaining species, B. laevis, morphologically similar to B. columnaris and B. procyonis (Berry, 1985; Anderson, 2000), occurring in large rodents as marmots and ground squirrels, and $B$. devosi occurring in martens and fishers, are transmissible in South American wildlife. A type material from these species is therefore needed to be subjected to further sequence analyses to better clarify the taxonomic issue in the JP1 isolate.

Vilas et al. (2005) appointed that because the ITS is a noncoding sequence, frequent deletions and insertions are often present making alignment more complicated. This was also the case of ribosomal data gathered in this study, with the preponderance of insertions in the examined isolate compared to the present Baylisascaris sequences. A varying number of $\mathrm{G}-\mathrm{A}$ tandem repeats can be used as discriminative marker for Baylisascaris spp. in the specific zone of the ITS2 region (positions 246 and further). Nine G-A tandem repeats were identified for B. procyonis, eight for $B$. transfuga, seven and six for the two B. columnaris genotypes, and one for $B$. schroederi that likely represents the ancestral state of the given DNA region (Testini et al., 2011; Lin et al., 2012; Franssen et al., 2013). For the JP1 isolate, 11 tandem contiguous G-A repeats were recorded in the respective locations. Two of them along with the additional interspersed motifs with $\mathrm{G}$, A bases have constituted 22-26 insertions compared to B. procyonis and $B$. columnaris, indicating a tendency towards lengthening the ITS2 region. The proposed mechanisms of evolution of repetitive sequences include both intra- or interstrand recombinational effects or mechanisms involving failures in the DNA replication (Platas et al., 2001). Mutational changes can create new motifs that may be propagated by additional slipped-strand mispairing (SSM) events. This was the plausible scenario for amplifying G-A repetitions in Baylisascaris spp., which formerly likely arose by chance in ITS2 and could have been further generated by SSM events into longer repeats.

Based on the gathered data, it is likely that the peculiar Baylisascaris species circulating in Guyanese zoofauna has been translocated to Japan via the infected kinkajou. Nevertheless, further characterization of Baylisascaris isolates from other kinkajou hosts is required, with particular emphasis on adult morphology and the pathogenicity of migrating larvae in paratenic hosts. In addition, to address potential limitations of single locus analyses, both nuclear and mtDNA regions will be targeted in molecular assays of Baylisascaris $\mathrm{sp}$. to facilitate species delineation.

Till now, there are over 20 documented cases of ocular larva migrans and severe or even fatal neurological disturbances in humans implied by B. procyonis (Galvin et al., 2005; Bauer, 2013). Zoonotic potential of B. columnaris is not yet known as serological assays do not discriminate between Baylisascaris species (Dangoudoubiyam et al., 2011). Experimental infections showed that $B$. procyonis is more pathogenic to mice than $B$. columnaris owing to faster growth to $1 \mathrm{~mm}$ size, which correlates with the first appearance of nervous symptoms (Tiner, 1953; Sheppard \& Kazakos, 1997). However, both species are along with B. melis (principal host badger) regarded as the most dangerous Baylisascaris members because of their serious disease-producing capabilities (Kazakos, 2001).

Given that the Baylisascaris sp. from the kinkajou is genetically closely affiliated with $B$. procyonis, the potential risk of human infection with Baylisascaris from this host may be considerable. Therefore, transmission-based precautions are needed to prevent human infections of pet kinkajous. It is strongly recommended that owners should take their kinkajous regularly to veterinarian for periodic fecal examination.

\section{Acknowledgements}

The authors wish to thank Dr. Ritsuko Hayashi at Momonoki Veterinary Clinic, Japan, for providing us with fecal and nematode samples. This work was partially supported by a Grant-in-Aid from Ministry of Health, Labor and Welfare, Japan and the Scientific Grant Agency VEGA (project No. 2/0172/13).

\section{References}

ANDERSON, R. C. (2000): Nematode parasites of vertebrates: Their development and transmission, 2nd ed. Wallingford, UK: CAB International, $650 \mathrm{pp}$.

BAUER, C. (2011): Baylisascariosis (Baylisascaris procyonis) - a rare parasitic zoonosis in Europe. Berl. Munch. Tierarztl. Wochenschr., 124: 465 - 472 (In German)

BAUER, C. (2013): Baylisascariosis - Infections of animals and humans with 'unusual' roundworms. Vet. Parasitol., 193: 404 - 412. DOI: 10.1016/ j.vetpar.2012.12.036

BERRY, J. F. (1985): Phylogenetic relationship between Baylisascaris spp. Sprent, 1968 (Nematoda: Ascarididae) from skunks, raccoons and groundhogs in southern Ontario. M. S Thesis. University of Guelph, Guelph, Ontario, Canada, 99 pp.

BLOUIN, M. S. (2002): Molecular prospecting for cryptic species of nematodes: mitochondrial DNA versus internal transcribed spacer. Int. J. Parasitol., 32: 527 - 531

Dangoudoubiyam, S., Vemulapalli, R., NDAO, M., KAZACOS, K. R. (2011): Recombinant antigen-based enzyme-linked immunosorbent assay for diagnosis of 
Baylisascaris procyonis larva migrans. Clin. Vaccine Immunol., 18: 1650 - 1655. DOI: 10.1128/CVI.00083-11 Derycke, S., De Ley, P., De Ley, I. T., Holovachov, O., RigauX, A., MoEns, T. (2010): Linking DNA sequences to morphology: cryptic diversity and population genetic structure in the marine nematode Thoracostoma trachygaster (Nematoda, Leptosomatidae). Zool. Scr., 39: 276 - 289. DOI: 10.1111/j.1463-6409.2009.00420.x

Despres, L., Imbert-Establet, D., Combes, C., BonHOMME, F. (1992): Molecular evidence linking hominid evolution to recent radiation of schistosomes (Platyhelminthes: Trematoda). Mol. Phylogenet. Evol., 1: 295 - 304 Ellis, R. E., Sulston, J. E., Coulson, A. R. (1986): The rDNA of C. elegans: sequence and structure. Nucleic Acids Res., 14: $2345-2364$

Franssen, F., Xie, K., Sprong, H., VAn der Giessen, J. (2013): Molecular analysis of Baylisascaris columnaris revealed mitochondrial and nuclear polymorphisms. Parasit. Vectors, 6: 124. DOI: 10.1186/1756-3305-6-124

Gavin, P. J., Kazacos, K. R., Shulman, S. T. (2005): Baylisascariasis. Clin. Microbiol. Rev. 18: $703-718$

Kazacos, K. R., Kilbane, T. P., Zimmerman, K. D., Chavez-Lindell, T., Parman, B., Carpenter, L. R., Green, A. L., Mann, P. M., Murphy, T. W., Bertucci, B., Gray, A. C., Goldsmith, T. L., Cunningham, M., StaneK, D. R., Blackmore, C., Yabsley, M. J., Montgomery, S. P., Bosserman, E. (2011): Raccoon roundworms in pet kinkajous--three states, 1999 and 2010. MMWR Morb. Mortal. Wkly. Rep., 60: 302 - 305

KAZACOS, K. R. (2001): Baylisascaris procyonis and related species. In: SAMUEL, W. M., PyBus, M. J., KocAn, A. A. (Eds) Parasitic diseases of wild animals., $2^{\text {nd }}$ ed. Iowa State University Press, London, pp. 301-341

MiYASHITA, M. (1993): Prevalence of Baylisascaris procyonis in raccoons in Japan and experimental infections of the worm to laboratory animals. J. Urban Living Health Assoc., 37: 137 - 151 (In Japanese)

LIN, Q., LI, H. M., GAO, M., WANG, X. Y., REN, W. X., CONG, M. M., TAN, X. C., ChEN, C. X., YU, S. K., ZHAO, G. H. (2012): Characterization of Baylisascaris schroederi from Qinling subspecies of giant panda in China by the first internal transcribed spacer (ITS-1) of nuclear ribosomal DNA. Parasitol. Res., 110: 1297 - 1303

Nadler, S. A., Hudspeth, D. S. (2000): Phylogeny of the Ascaridoidea (Nematoda: Ascaridida) based on three genes and morphology: hypotheses of structural and sequence evolution. J. Parasitol., 86: 380 - 393

OverstreEt, B. M. (1970): Baylisascaris procyonis (Stefanski and Zarnowski, 1951) from the kinkajou, Potos flavus, in Colombia. Proc. Helminthol. Soc., 32: 192 - 195 PaWAR, R. M., LAKShMiKantan, U., Hasan, S., PoORNACHANDAR, A., SHIVAJI, S. (2012): Detection and mole- cular characterization of ascarid nematode infection (Toxascaris leonina and Toxocara cati) in captive Asiatic lions (Panthera leo persica). Acta Parasitol., 57: 67 - 73. DOI: 10.2478/s11686-012-0012-y

Platas, G., ACERO, J., Borkowski, J. A., GonzÁlez, V., Portal, M. A., Rubio, V., SÁnchez-BAllesteros, J., SALAZAR, O., PELÁEZ, F. (2001): Presence of a simple tandem repeat in the ITS1 region of the Xylariales. Curr. Microbiol., 43: 43 - 50

Qu, L. H., Nicoloso, M., BAChellerie, J. P. (1988): Phylogenetic calibration of the $5^{\prime}$ terminal domain of large rRNA achieved by determining twenty eucaryotic sequences. J. Mol. Evol., 28: 113 - 124

RoepstorfF, A., NANSEn, P. (1998): The epidemiology, diagnosis and control of helminth parasites of swine. FAO Animal Health Manual No. 3, FAO, Rome, Italy.

SATO, H., FURUOKA, H., KAMIYA, H. (2001): First outbreak of Baylisascaris procyonis larva migrans in rabbits in Japan. Parasitol. Int., 51: $105-108$

Sheppard, C. H., KazAcos, K. R. (1997): Susceptibility of Peromyscus leucopus and Mus musculus to infection with Baylisascaris procyonis. J. Parasitol., 83: $1104-1111$ ŠnÁBEl, V., TAIRA, K., CAVAllero, S., D’Amelio, S., RUdOHRADSKÁ, P., SAITOH, Y. (2012): Genetic structure of Ascaris roundworm in Japan and patterns of its geographical variation. Jpn. J. Infect. Dis., 65: $179-183$

Tamura, K., Peterson, D., Peterson N., Stecher, G., NEI, M. (2011): MEGA5: Molecular Evolutionary Genetics Analysis Using Maximum Likelihood, Evolutionary Distance, and Maximum Parsimony Methods. Mol. Biol. Evol., 28: 2731 - 2739. DOI: 10.1093/molbev/msr121

Testini, G., Papini, R., Lia, R. P., PARisi, A., DAntasTorres, F., Traversa, D., Otranto, D. (2011): New insights into the morphology, molecular characterization and identification of Baylisascaris transfuga (Ascaridida, Ascarididae). Vet. Parasitol., 175: 97 - 102. DOI: 10.1016/j.vetpar.2010.09.017

Thompson, J. D., Higgins, D. G., Gibson, T. J. (1994): Clustal W: Improving the sensitivity of progressive multiple sequence alignment through sequence weighting, position-specific gap penalties and weight matrix choice. $\mathrm{Nu}$ cleic Acids Res., 22: 4673 - 4680

Vilas, R., CRiscione, C. D., Blouin, M. S. (2005): A comparison between mitochondrial DNA and the ribosomal internal transcribed regions in prospecting for cryptic species of platyhelminth parasites. Parasitology, 131: 839 $-846$

Zhu, X. Q., Gasser, R. B., Chilton, N. B., Jacobs, D. E. (2001): Molecular approaches for studying ascaridoid nematodes with zoonotic potential, with an emphasis on Toxocara species. J. Helminthol., $101-108$ 